10.2

\title{
Экспериментальное исследование многополосной акустооптической фильтрации при декодировании спектрально-кодированных сигналов в некогерентных системах OCDMA
}

\author{
(C) А.Л. Филатов \\ Фрязинский филиал Института радиотехники и электроники им. В.А. Котельникова РАН, Фрязино, Московская обл., \\ Россия \\ E-mail: a.filatov@fireras.su
}

Поступило в Редакцию 22 июня 2020г.

В окончательной редакции 18 сентября 2020г.

Принято к публикации 18 сентября 2020г.

\begin{abstract}
Экспериментально доказана возможность использования акустооптических многополосных фильтров для построения сетей с кодовым разделением каналов множественного доступа. Исследованы корреляционные помехи, вызванные тем, что для кодирования/декодирования используется набор полос, спектр которых имеет вид, близкий к функции $\sin (x) / x$. Для случая нескольких передатчиков разработана и реализована специальная технология, позволившая провести исследования на линии передач, созданной на базе двух фильтров. Ключевой составляющей технологии является эмулирование на первом фильтре суммарного сигнала от двух-пяти передатчиков. Измеренная величина отношения амплитуд сигнала к интерференционным помехам была больше $11 \mathrm{~dB}$ и уменьшалась из-за корреляционных помех от сетевого окружения и ошибки эмулирования суммарного сигнала менее чем на $3 \mathrm{~dB}$.
\end{abstract}

Ключевые слова: акустооптический фильтр, спектральное кодирование, DDS, OCDMA.

DOI: 10.21883/PJTF.2021.01.50452.18432

Технология оптической связи с кодовым разделением каналов множественного доступа OCDMA (optical code division multiple access) в отличие от различных вариантов реализации технологий WDM (wavelength-division multiplexing) и TDMA (time division multiple access), в которых каналы разделяются по длинам волн и времени, позволяет не только эффективно использовать пропускную способность оптического волокна, но и аппаратно реализовать защиту передаваемой информации [1]. В источниках по тематике OCDMA [2,3] отсутствует информация об использовании в таких системах акустооптических фильтров (АОФ), которые потенциально позволяют реализовать конкурентоспособные с WDM простые коммерческие решения, описанные, например, в [4]. Это, скорее всего, вызвано тем, что продемонстрированное [4] в прошлом веке низкое качество кодирования и отсутствие экспериментов по декодированию, для которых необходима работа АОФ в многочастотном режиме, указывало на бесперспективность применения акустооптических технологий в сетях OCDMA.

Идея использования многочастотного режима для расширения функционала всех акустооптических (AО) устройств неоднократно предлагалась в публикациях ведущих специалистов $[5,6]$ и была аппаратно реализована за счет использования в одной АО-ячейке нескольких пьезопреобразователей [7]. Об отсутствии стандартной реализации такого режима свидетельствует то, что все современные серийные АОФ используют одночастотный акустический сигнал. В последнее время благодаря прогрессу цифровых технологий была экс- периментально подтверждена эффективность двух подходов к решению проблемы многочастотного режима в АО-устройствах. Первый подход, развивающий идеи, похожие на рекомендации из работы [4], был впервые экспериментально апробирован в [8] и продолжает изучаться теоретически $[9,10]$. Эффективность второго подхода, в котором для формирования акустического сигнала используются цифровые синтезаторы частоты (direct digital synthesizers, DDS), была экспериментально продемонстрирована в трех приложениях: 1) для подавления нулевого порядка акустооптической дифракции сфокусированного лазерного излучения [11]; 2) для акустооптического управления энергетическим профилем лазерного излучения [12]; 3) для спектрального кодирования некогерентного излучения [13].

Инновации в технологиях создания форм спектров пропускания АОФ инициировали проведение серии исследований, конечной целью которых является получение экспериментального подтверждения возможности использования АОФ в системах ОСDMА. В [14] были переосмыслены предложенные в работе [4] схемотехнические решения построения сетей OCDMA, которые должны позволить реализовать скорость передачи до $16 \mathrm{Gbit} / \mathrm{s}$; показано, как можно выполнить бинарную передачу данных путем тактированного переключения между сопряженными функциями Уолша, реализованными в виде набора полос, спектр которых имеет вид, близкий к функции $\sin (x) / x$; проведено, возможно, некорректное моделирование зависимости амплитуды корреляционных помех от шага сетки и ширины полос. 
На эту некорректность указывает то, что амплитуда помех на рис. 3 из работы [14] растет при бесконечном увеличении спектрального расстояния между соседними полосами, спектральная ширина которых остается постоянной. В [13] было получено принципиально лучшее, чем в [4], качество амплитудного кодирования излучения светодиода функциями Уолша. В [15] на линии передач, созданной на базе двух АОФ, были проведены и описаны эксперименты по кодированию/декодированию сигналов от одного передатчика, которые формируются функциями Уолша.

В настоящей работе исследовались связанные со спектральной формой сигнала корреляционные помехи, возникающие при работе двух-пяти передатчиков в такой же линии передач и с таким же набором отстоящих друг от друга на $\sim 9.5 \mathrm{~nm}$ спектральных полос с полушириной $\sim 1.6 \mathrm{~nm}$, как в [15]. В качестве характеристики шума сети, как и в [15], было выбрано отношение амплитуд сигнала к интерференционным помехам (signal to interference ratio, SIR), которое определялось без учета шума передаваемого сигнала. Отношение сигнала к суммарному шуму (signal to interference + noise ratio, SINR) необходимо учитывать для оценки скорости передачи. Чтобы усредненный шум сигнала был заведомо меньше, чем шум каналов сети, интенсивность света и спектры пропускания АОФ в ходе каждого эксперимента не изменялись в течение $10 \mathrm{~s}$.

Первоначально планировалось в автоматическом режиме провести $10 \sum_{n=1}^{5} 2^{n} C_{5}^{n}=2420$ измерений в сети OCDMA, построенной на базе сопряженных с волокном АОФ. Однако в связи с невозможностью создания волоконной сети из-за прекращения внебюджетного финансирования было реализовано паллиативное решение, основанное на эмулировании суммарного сигнала от двух-пяти передатчиков на одном АОФ. Математическое моделирование величин SIR позволило уменьшить необходимое количество экспериментов с 2420 до 260. При моделировании использовались показанные на рис. 1 экспериментальные спектры, которые соответствуют всем возможным пяти взаимно комплементарным последовательностям функций Уолша. Эти спектры были получены на базе частотно-эквидистантной сетки от 79.0 до $84.6 \mathrm{MHz}$ с шагом $0.8 \mathrm{MHz}$, которую можно легко пересчитать в спектральную исходя из соотношения $\lambda f=$ const, где $\lambda$ - длина волны света, $f-$ частота акустического сигнала. В используемых АОФ const $\approx 76 \mathrm{MHz} \cdot \mu \mathrm{m}$.

В ходе моделирования для экспериментальной проверки качества эмуляции суммарного сигнала было выбрано восемь спектров с минимальными помехами от сетевого окружения $(|\Delta \mathrm{SIR}|<1 \mathrm{~dB})$. Для изучения максимального влияния сетевого окружения на корреляционные помехи была сформирована состоящая из восьми спектров выборка с максимальными помехами $(\mathrm{SIR} \approx 12 \mathrm{~dB})$. К двум выборкам была добавлена третья из десяти спектров, которая исследовалась в [15].

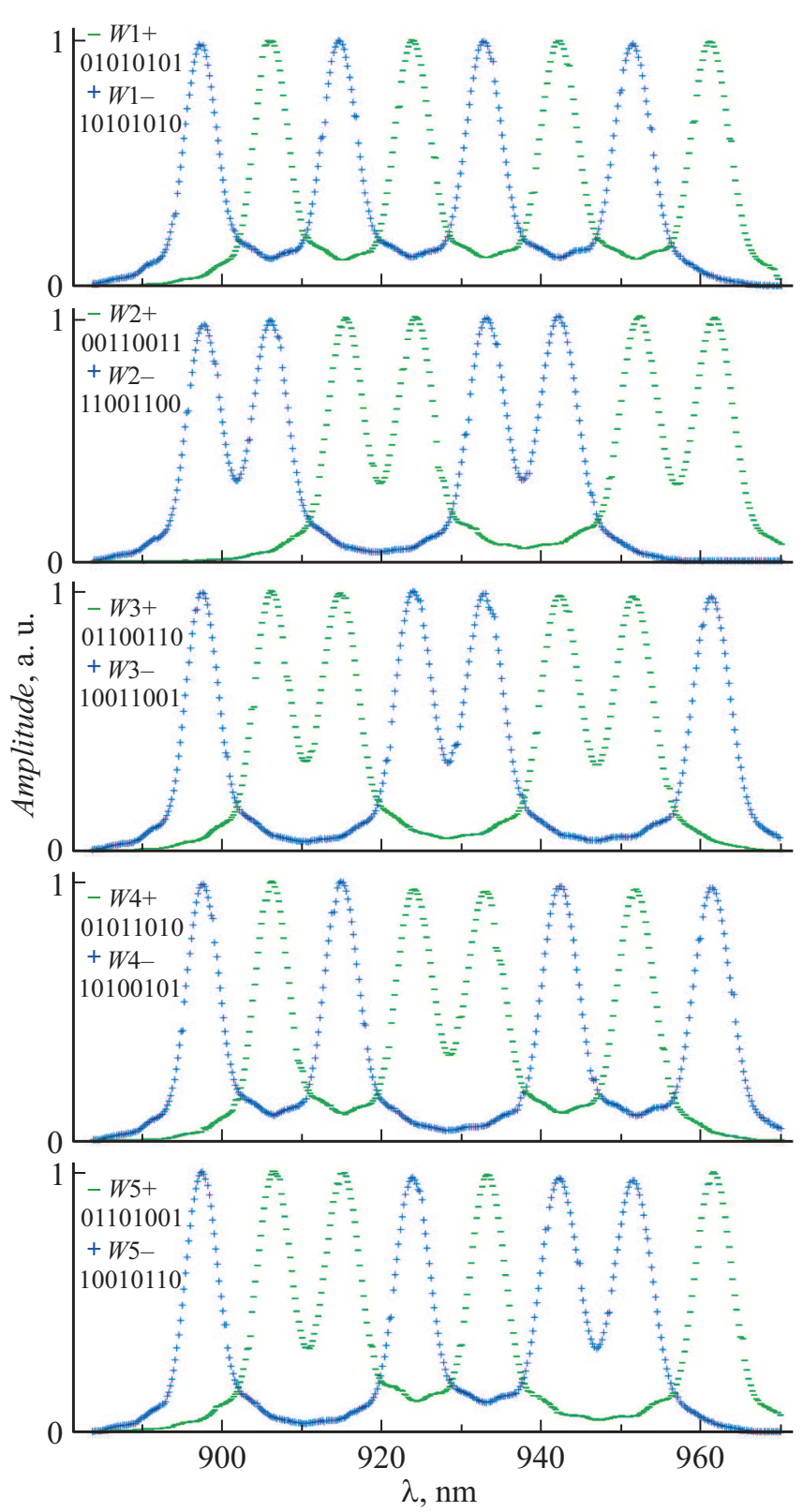

Рис. 1. Спектры излучения от LED, кодированные первым АОФ, соответствующие взаимно комплементарным последовательностям Уолша. Измерения выполнены при помощи второго АОФ, работающего в режиме перестраиваемого фильтра.

В ходе экспериментов каждый спектр (сигнал) декодировался с использованием десяти спектров, показанных на рис. 1. При исследовании первой выборки было определено, что суммарные ошибки эмуляции и интерференционные помехи от сетевого окружения привели к незначительному увеличению шума $|\Delta \mathrm{SIR}|<3 \mathrm{~dB}$. Это доказывает корректность эмуляции суммарного сигнала. Для иллюстрации качества эмуляции на рис. 2 показаны полученные двумя способами спектры, соответствующие одновременной работе пяти передатчиков. Сплошная линия соответствует сумме ранее выполненных спектральных измерений, которые представлены 


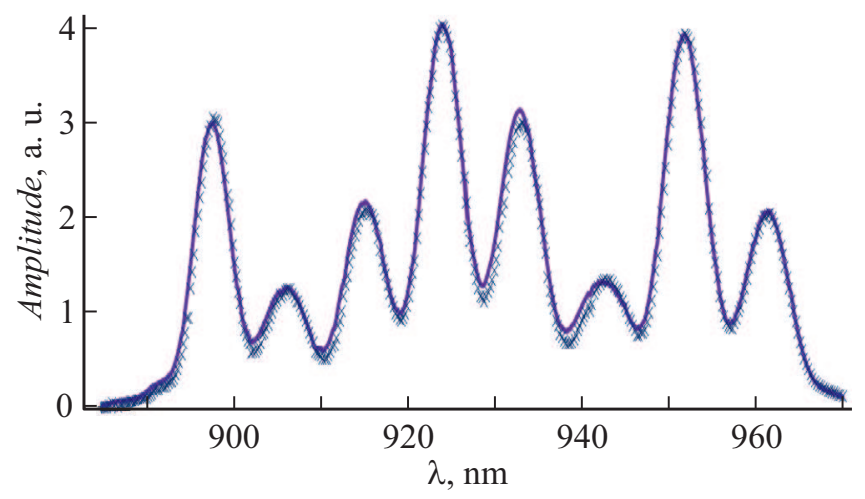

Рис. 2. Спектр, эмулирующий суммарный сигнал от пяти передатчиков $\sum(W 1-, W 2+, W 3-, W 4+, W 5-)$, которому соответствует код Уолша (31243142). Точки соответствуют прямым экспериментальным измерениям, сплошная линия сумме пяти ранее выполненных спектральных измерений (см. рис. 1).

на рис. 1. Точками показаны результаты экспериментальных измерений эмулированного суммарного сигнала. Видно хорошее количественное совпадение обеих спектральных зависимостей. Для второй выборки, соответствующей сети с максимальным корреляционным шумом от нескольких передатчиков, было определено, что $\mathrm{SIR}>11 \mathrm{~dB}$. Это хороший результат с учетом того, что $\mathrm{SINR}>10 \mathrm{~dB}$ достаточен для стабильной работы сети. Данные исследования третьей выборки, отвечающей работе одного передатчика вне сетевого окружения, совпадают с результатами [15]: SIR > $13 \mathrm{~dB}$. Видно, что помехи от сетевого окружения и ошибки эмулирования суммарного сигнала ухудшили SIR менее чем на $3 \mathrm{~dB}$.

Таким образом, впервые была экспериментально показана возможность использования АОФ, работающих под управлением DDS-генераторов, для эмуляции спектра пропускания суммарного сигнала от двух-пяти передатчиков, которые спектрально кодированы функциями Уолша. Впервые в построенной на базе акустооптических фильтров сети ОСDMА экспериментально исследованы систематические корреляционные помехи, вызванные тем, что для кодирования/декодирования используется набор полос, спектр которых имеет вид, близкий к функции $\sin (x) / x$. Полученные результаты с учетом данных [13-15] экспериментально доказывают возможность и перспективность применения многополосной акустооптической фильтрации в безволоконных некогерентных системах OCDMA.

\section{Благодарности}

Автор благодарит В.М. Епихина за предоставление эскизов согласованной пары АОФ.

\section{Финансирование работы}

Работа выполнена в рамках государственного задания.

\section{Конфликт интересов}

Автор заявляет, что у него нет конфликта интересов.

\section{Список литературы}

[1] K.-S. Chen, W. Hong, Appl. Sci., 10, 730 (2020). DOI: 10.3390app10030730

[2] Р. Фриман, Волоконно-оптические системы связи, пер. с англ. под ред. Н.Н. Слепова (Техносфера, М., 2003), с. 447.

[3] H. Yin, D.J. Richardson, Optical code division multiple access communication networks: theory and applications (SpringerVerlag, Berlin-Heidelberg, 2007).

[4] O. Ziemann, K. Iversen, Proc. SPIE, 2614, 143 (1995). DOI: $10.1117 / 12.227825$

[5] В.Б. Волошинов, В.Н. Парыгин, Б. Траоре, Вестн. Моск. ун-та. Сер. 3. Физика, астрономия, № 5, 50 (1988).

[6] В.Э. Пожар, В.И. Пустовойт, Изв. РАН. Сер. физ., 79 (10), 1375 (2015).

[7] В.И. Пустовойт, В.Э. Пожар, Вестн. МГТУ им. Н.Э. Баумана. Сер. Приборостроение, № 52, 6 (2011).

[8] С.Н. Антонов, ЖТФ, 75 (4), 122 (2005).

[9] С.Н. Антонов, Ю.Г. Резвов, Радиотехника и электроника, 50 (4), 472 (2005).

[10] В.В. Проклов, Ю.Г. Резвов, Оптика и спектроскопия, 124 (1), 122 (2018).

[11] А.Л. Филатов, В сб. VII Межсдунар. конф. по фотонике и информатике (НИЯУ „МИФИ“, М., 2018), с. 85.

[12] С.Н. Антонов, А.Л. Филатов, ЖТФ, 88 (1), 93 (2018).

[13] В.В. Проклов, О.А.Бышевский-Конопко, А.Л.Филатов, Письма в ЖТФ, 41 (20), 37 (2015).

[14] В.В. Проклов, О.А. Бышевский-Конопко, В.И. Григорьевский, Квантовая электроника, 43 (6), 542 (2013).

[15] V.V. Proklov, O.A. Byshevski-Konopko, A.L. Filatov, A.V. Lugovskoi, Y.V. Pisarevsky, J. Phys.: Conf. Ser., 737, 012060 (2016). DOI: 10.1088/1742-6596/737/1/012060 